\title{
Premotor activations in response to visually presented single letters depend on the hand used to write: a study on left-handers
}

\author{
Marieke Longcamp ${ }^{\mathrm{a}}$, Jean-Luc Anton ${ }^{\mathrm{b}}$, Muriel Roth ${ }^{\mathrm{b}}$, Jean-Luc Velay ${ }^{\mathrm{a}, *}$ \\ ${ }^{a}$ The Mediterranean Institute for Cognitive Neuroscience (INCM), CNRS, 31 Chemin Joseph Aiguier, 13402 Marseille Cedex 20, France \\ b fMRI Center, CHU, 'La Timone' Hospital, Marseille, France
}

Received 8 October 2004; received in revised form 18 January 2005; accepted 27 January 2005

Available online 14 March 2005

\begin{abstract}
In a previous fMRI study on right-handers (Rhrs), we reported that part of the left ventral premotor cortex (BA6) was activated when alphabetical characters were passively observed and that the same region was also involved in handwriting [Longcamp, M., Anton, J. L., Roth, M., \& Velay, J. L. (2003). Visual presentation of single letters activates a premotor area involved in writing. NeuroImage, 19, 1492-1500]. We therefore suggested that letter-viewing may induce automatic involvement of handwriting movements. In the present study, in order to confirm this hypothesis, we carried out a similar fMRI experiment on a group of left-handed subjects (Lhrs). We reasoned that if the above assumption was correct, visual perception of letters by Lhrs might automatically activate cortical motor areas coding for left-handed writing movements, i.e., areas located in the right hemisphere. The visual stimuli used here were either single letters, single pseudoletters, or a control stimulus. The subjects were asked to watch these stimuli attentively, and no response was required. The results showed that a ventral premotor cortical area (BA6) in the right hemisphere was specifically activated when Lhrs looked at letters and not at pseudoletters. This right area was symmetrically located with respect to the left one activated under the same circumstances in Rhrs. This finding supports the hypothesis that visual perception of written language evokes covert motor processes. In addition, a bilateral area, also located in the premotor cortex (BA6), but more ventrally and medially, was found to be activated in response to both letters and pseudoletters. This premotor region, which was not activated correspondingly in Rhrs, might be involved in the processing of graphic stimuli, whatever their degree of familiarity.
\end{abstract}

(C) 2005 Elsevier Ltd. All rights reserved.

Keywords: Handedness; fMRI; Handwriting; Perceptual-motor interactions; Letter perception; Premotor cortex

\section{Introduction}

According to the motor theory of perception, perceptual processes are influenced and guided by the perceiver's own repertory of potential gestures (for a review, see Viviani \& Stucchi, 1992). One of the assumptions made by motor theorists about the perception of space and objects is that motor-perceptual interactions are strongly determined by the way subjects interact within their environment (Paillard, 1991). One particular example of a strong association between a gesture and a category of objects is that between handwriting and graphic forms. Since we know how to write,

\footnotetext{
* Corresponding author. Tel.: +33 4911642 39; fax: +33 491164296 . E-mail address: velay@ incm.cnrs-mrs.fr (J.-L. Velay).
}

each character we know is coded in memory under the form of a specific motor program (van Galen, 1991), and we might automatically refer to these writing programs even when we are simply looking at characters. If so, the nature of the motor-perceptual interactions involved in reading is likely to depend on the way we write: somebody who knows how to read but doesn't know how to write should not be able to associate the visual form of a character with any specific motor process. Someone who has learned how to write and usually writes with his/her right hand will probably associate a character with motor schemes specific to the right hand, whereas someone who has learned how to write and usually writes with his/her left hand will make use of motor schemes specific to the left hand. The present experiment was designed to test this idea. 
Several empirical findings support the hypothesis that motoric writing knowledge contributes automatically to the processing of visually perceived characters. From this viewpoint, Chinese and Japanese ideograms are striking examples. In these graphic systems, each character is composed of a number of strokes that must be written in a precise order when learning to read and write. The order of the strokes is subsequently used as a cue to retrieve the ideograms from memory (Flores d'Arcais, 1994), suggesting that the motor sequence of strokes specific to each ideogram may be an essential component of its central representation. Kanji ideogram retrieval has been found to activate several brain regions usually involved in handwriting (Kato et al., 1999). In alphabetical systems, a comparable example is provided by a case study by Anderson, Damasio, and Damasio (1990). The patient in question became agraphic as the result of a left premotor cortical lesion. The fact that her inability to write was associated with a specific visual letter identification deficit shows that the lack of the motor programs for writing can prevent subjects from being able to recognise letters and consequently, from reading.

In a previous neuroimaging study (Longcamp, Anton, Roth, \& Velay, 2003), we directly assessed the possibility that motoric writing skills might be automatically involved in the visual perception of alphabetical letters. Using fMRI on a group of right-handed subjects (Rhrs), we checked whether passive letter-viewing induced any activation in the sensorimotor brain areas known to be involved in writing movements. We observed that part of the left ventral premotor cortex (Brodmann area 6) was activated when alphabetical characters were being passively observed and that the same region was also involved in handwriting. Interestingly, this area did not respond to the visual presentation of pseudoletters, to which no predetermined motor program could be associated. We therefore suggested that the premotor activation observed reflected the involvement of the motor programs for writing, corresponding to each letter, in agreement with the conclusions drawn by Anderson et al. (1990). Although our experimental results strongly support this conclusion, the premotor activation can also be explained in terms of linguistic processing. Subvocal articulatory processes are known to activate higher order motor areas, especially during silent or implicit reading (Price et al., 1994; Price, Wise, \& Frackowiak, 1996) and working memory tasks (Chen, Cohen, \& Hallet, 1997; Paulesu, Frith, \& Frackowiak, 1993; Smith \& Jonides, 1999).

In the present study, we were looking for stronger evidence in favor of our hypothesis. We therefore repeated the experiments previously carried out on Rhrs with a group of left-handers (Lhrs) who consistently used their left hand to write. One of the main characteristics of writing movements is their high degree of lateralization and dependence on handedness, whereas language and spatial abilities are thought to be more widely distributed over the whole brain in Lhrs. Although the cerebral organization of Lhrs is still a matter of discussion, it seems likely that, as in Rhrs, language is pre- dominantly processed by the left hemisphere in a majority of Lhrs (Hécaen \& Sauguet, 1971). For instance, in a recent study using functional transcranial Doppler ultrasonography, a perfusion-sensitive technique, Knecht et al. (2000) measured the incidence of right-hemispheric dominance for language in a phonological word generation task. These authors reported that in a sample of 326 individuals, the incidence of right-hemispheric language dominance increased with the degree of left-handedness from $4 \%$ in strong Rhrs (handedness $=100$ in terms of the Edinburgh handedness inventory score (Oldfield, 1971)), to $15 \%$ in ambidextrous individuals and $27 \%$ in the strongest Lhrs (handedness $=-100$ ). The assumption about the localization of writing engrams in Lhrs requires some discussion, however although it is generally recognised that motor programs for writing are leftlateralized in most Rhrs (Katanoda, Yoshikawa, \& Sugishita, 2001; Menon \& Desmond, 2001; Rijntjes et al., 1999), since both their language and motor skills are controlled by the left hemisphere, the situation as far as Lhrs are concerned is less clear-cut and has been less well documented. Nonetheless, there exist several grounds for presuming that graphic motor representations are entirely right-lateralised in these subjects. First, according to Rijntjes et al. (1999), who examined the cerebral zones involved in signing, highly trained movements of this kind may be stored in the same brain areas as those controlling the movements of the limb ordinarily used for their execution (i.e., in the right hemisphere in Lhrs). Secondly, Herron, Galin, Johnstone, and Ornstein (1979) measured EEG asymmetry in handwriting as compared to other linguistic tasks such as listening to a story, relating it or reading it. The right central region was found to be consistently involved during handwriting in those subjects who usually wrote with their left hand, regardless of their pattern of lateralization for language and other motor abilities. Thirdly, studies on patients with apraxic agraphia, a disorder affecting motor aspects of writing but sparing other language functions (Roeltgen, 1985), have also provided some information about the lateralization of the neural basis of handwriting. For instance, Margolin (1980) described a fully left-handed patient who became apraxic and agraphic as the result of a right central lesion, but showed no associated language deficits. On similar lines, Margolin and Binder (1984) reported the case of a patient who wrote with his left hand and was mixed-handed when performing other activities. After having a right hemispheric stroke, he presented with apraxic agraphia without any limb-motor apraxia or aphasia. These cases point to the fact that motor programs for writing may be separate from other language and motor functions and suggest that they are probably set up in the hemisphere contralateral to the usual writing hand. Finally, a more direct piece of evidence comes from a study by Siebner et al. (2002) who found an exclusive activation of the right sensorimotor cortex when a group of Lhrs was performing a simple handwriting task.

On the basis of these data, we reasoned that if our previous interpretation was true, then there would be only one possible pattern of results for Lhrs: visual perception of sin- 
gle letters would automatically activate cortical motor areas located in the right hemisphere. Any other pattern of results (ie bilateral or left-sided activation) would have challenged our interpretation.

As was the case in comparable experiments that investigated the neural correlates of perception of manipulable objects in both monkeys (Murata et al., 1997) and humans (Chao \& Martin, 2000), stimuli were presented in the context of passive perception. There were two main reasons for this choice: first, we wanted to prevent any interactions from occurring between the implicit motor activations of interest and the explicit motor processes involved in a manual response (Friston et al., 1996, and see Hanakawa et al., 2002 for a discussion of that issue). Second, we wanted to prevent systematic involvement of two particular cognitive processes: visuospatial attention in a feature-decision task and phonological processes in a linguistic task. Both are likely to automatically activate some motor brain areas.

\section{Methods}

\subsection{Subjects and experimental design}

The study was carried out on 13 healthy adult native French volunteers ( 9 males and 4 females, mean age $27 \pm 5$ years). The main criterion on which inclusion was based was the consistent use of the left hand for writing: this point was checked when interviewing the subjects prior to the experiment. However, in order to ensure that the sample of subjects tested was homogeneous, manual laterality was assessed based on the Edinburgh handedness inventory (Oldfield, 1971) and we included only those subjects who used their left hand preferentially to perform everyday activities. The handedness scores ranged from -50 to -100 (mean -78). Participants were free from neurological diseases and any history of dyslexia or other language disability. They all gave their informed consent and the study was approved by the local ethics committee.

The visual stimuli were either single letters, single pseudoletters, or a control stimulus (Fig. 1). The subjects were simply asked to look at these stimuli attentively, and no response was required. To prevent any mental writing strategy, we did not specify the exact aim of the study to the subjects before the scanning sessions. The visual display was generated on a PC screen and projected onto a rear projection screen located $60 \mathrm{~cm}$ from the subject's eyes in the magnet. The subjects viewed the screen in a darkened room via a mirror. There were three imaging sessions, each consisting of 15 epochs (5 "letter", 5 "pseudoletter", and 5 "control" epochs) presented in a pseudo-randomized order.

\subsection{Data acquisition and analysis}

Images were acquired on a 3-T MEDSPEC 30/80 AVANCE whole-body imager (Bruker, Ettlingen, Germany)

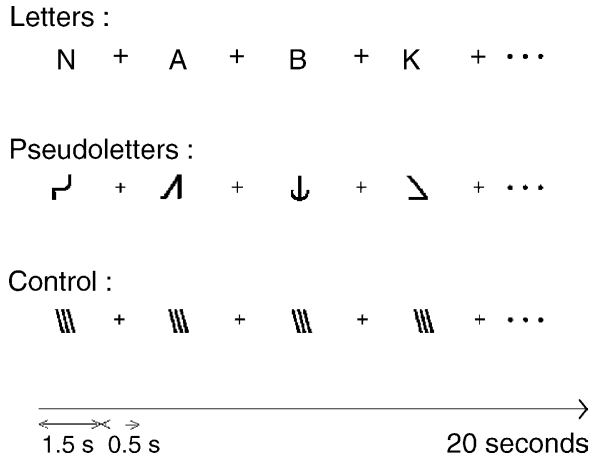

Fig. 1. Stimulus sequence. The three types of stimuli were presented in epochs consisting of 10 repetitions, each epoch lasting for $20 \mathrm{~s}$. The rate of presentation was 1 per $2 \mathrm{~s}(0.5 \mathrm{~s}$ fixation $+1.5 \mathrm{~s}$ display $)$. The control stimulus consisted of three oblique lines. The pseudoletters and the control stimulus were designed to match the letters visually in terms of their overall size and the mean number and thickness of strokes. To prevent unwanted eye movements from occurring, the stimuli were presented centrally on the screen and covered a visual angle of less than $1^{\circ}$. They were preceded by the presentation of a central fixation cross-lasting for $0.5 \mathrm{~s}$

equipped with a circular polarized head coil. We first acquired a high-resolution structural T1-weighted image (inversion-recovery sequence, $1 \mathrm{~mm} \times 0.75 \mathrm{~mm} \times 1.22 \mathrm{~mm}$ ) parallel to the AC-PC plane, covering the whole brain of each participant. For functional imaging, we used a T $2^{*}$-weighted echo-planar sequence covering the whole brain with 30 interleaved 3.5-mm-thick/1 mm-gap axial slices (repetition time $=2500 \mathrm{~ms}$; echo time $=35 \mathrm{~ms}$; flip angle $=80^{\circ}$; field of view $=19.2 \mathrm{~cm} \times 19.2 \mathrm{~cm} ; 64 \times 64$ matrix of $3 \mathrm{~mm} \times 3 \mathrm{~mm}$ voxels). We acquired 132 functional volumes per session.

Data were processed using spm99 software (http://www. fil.ion.ucl.ac.uk/spm/), according to the general linear model (Friston et al., 1995). The first four functional volumes of each session were removed to eliminate any non-equilibrium effects of magnetization. The remaining 128 images were corrected to compensate for differences in the slice acquisition time, and after this step we also discarded the first and last two volumes to prevent invalid temporal interpolation. The images were then corrected for head movement by realigning all the images with the first image using rigid body transformations normalized by matching them with the standardized MNI EPI-template (thus yielding an isotropic voxel size of $2 \mathrm{~mm}$ ), and spatially smoothed with an isotropic Gaussian filter ( $6 \mathrm{~mm}$ full width at half maximum).

At the first level of the statistical analysis, we modeled individual data by building a fixed-effects model where individual fMRI time-series were correlated with boxcar functions convolved with the Hemodynamic Response Function. In the temporal domain, we applied high-pass filtering with an $80 \mathrm{~s}$ cutoff and temporal smoothing with a $4 \mathrm{~s}$ Gaussian kernel. We assessed three linear contrasts per subject, namely letter versus control, pseudoletter versus control, and letter versus pseudoletter. The second level group study was based on a random effects model to account for the between-subject 
variance, and to allow inference at the population level. The summary scans of the 13 subjects were computed separately in three one-sample-t-tests, one for each of the three comparisons performed. We thresholded the statistical maps at $p \leq 0.001$, uncorrected for multiple comparisons at the voxel level. Significant clusters were localized using a standard stereotaxic anatomical brain atlas (Talairach \& Tournoux, 1988). In the tables, we report the Talairach coordinates, converted from the MNI coordinates using the mni2tal script provided at http://www.wjh.harvard.edu/ slotnick/scripts.htm.

\section{Results}

Only clusters consisting of 20 or more contiguous voxels were taken into account.

\subsection{Brain areas specifically activated by letters}

The results of the $t$-test performed on the letter versus pseudoletter contrast reflect the specific processing of the letter stimuli. The results of this comparison are given in Fig. 2 and Table 1. It is worth noting that this contrast elicited an activation in the precentral gyrus (BA 6) in the right hemisphere alone. This area may be located in the human equivalent of the superior part of the monkey ventral premotor cortex (Rizzolatti, Fogassi, \& Gallese, 2002). It will be referred to here as vPMCsup. In addition to this activation of particular interest, we found significant clusters in several
Table 1

Anatomical location, corresponding Brodmann area, lateralization, Talairach coordinates and $Z$-score for the activations specific to letters as assessed by the letters vs. pseudoletters contrast

\begin{tabular}{llrrl}
\hline Anatomical location & \multicolumn{3}{c}{ Coordinates $(\mathrm{mm})$} & Maximum Z-score \\
\cline { 2 - 4 } & $x$ & \multicolumn{1}{c}{$y$} & \multicolumn{1}{c}{$z$} & \\
\hline GFi (BA47) R & 32 & 17 & -13 & 4.94 \\
LPi (BA40) R & 61 & -41 & 32 & 4.62 \\
LPi (BA40) R & 51 & -51 & 41 & 4.29 \\
GPrC (BA 6) R & 51 & -2 & 39 & 4.11 \\
GTm (BA21) R & 59 & -28 & -10 & 4.02 \\
\hline
\end{tabular}

Abbreviations: $\mathrm{GFi}$, inferior frontal gyrus; $\mathrm{LPi}$, inferior parietal lobule; GPrC, precentral gyrus; GTm, middle temporal gyrus.

right hemispheric areas, in the parietal, inferior frontal and temporal cortices.

\subsection{Brain areas commonly activated in response to letters and pseudoletters}

The brain areas activated by both letter and pseudoletter perception are given in Fig. 3 and Table 2. They were assessed by masking inclusively the results of the $t$-tests performed on the letter versus control and pseudoletter versus control contrasts at a threshold of $p \leq 0.001$. The results obtained reflect the activations associated with the processing of any graphic stimulus, whatever its degree of familiarity. Among the areas activated, a bilateral effect was observed in the precentral gyrus (area 6, premotor cortex) in response to both passive letter and pseudoletter viewing, in addition to several bilat-
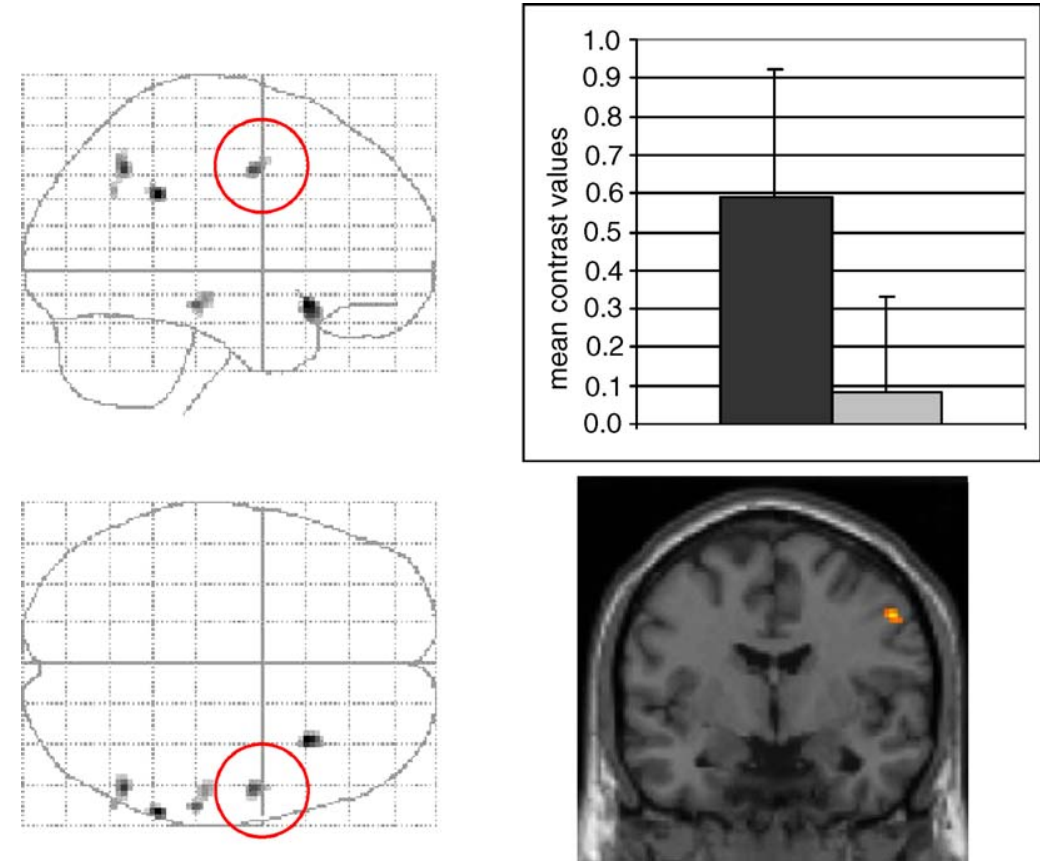

Fig. 2. Patterns of activation observed in the letter vs. pseudoletter comparison. On the left, "glass brain" views: the activation occurring in the right precentral gyrus (vPMCsup) is circled in red. On the bottom right, the same pattern of activation is shown on a coronal brain slice $(y=-2)$. On the top right, the mean contrast values within this region for the letters vs. control (black) and pseudoletters vs. control (grey) contrasts are displayed (errors bars denote the confidence intervals at 95\%). (For interpretation of the references to colour in this figure legend, the reader is referred to the web version of the article.) 


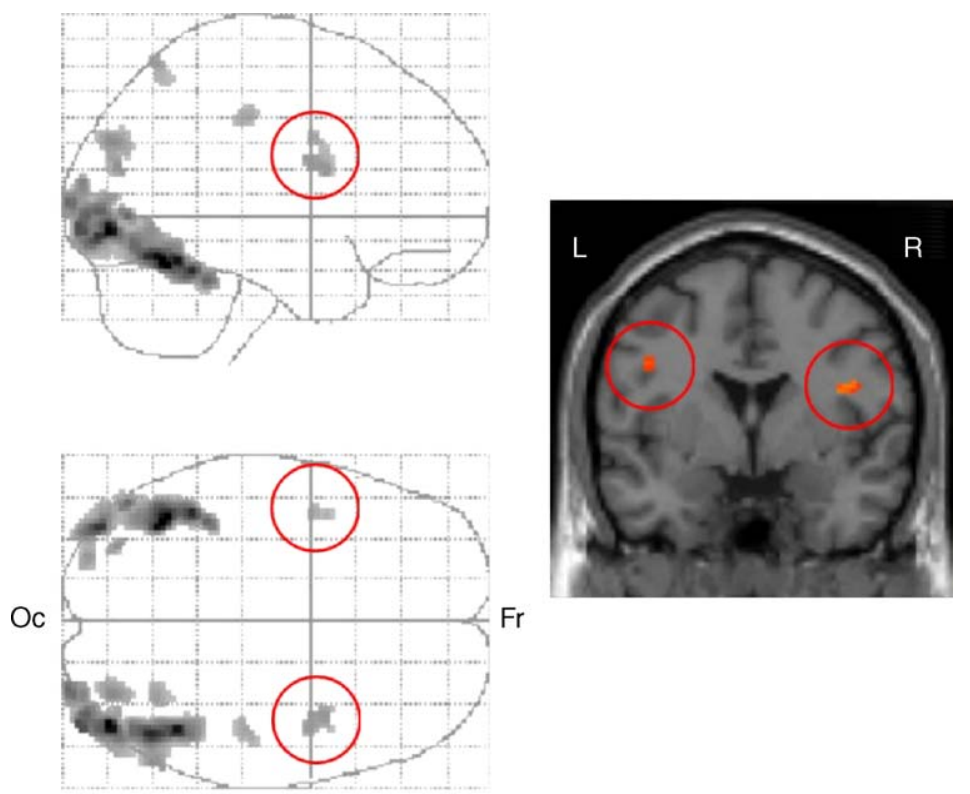

Fig. 3. Patterns of activation observed in the inclusive masking of the letter vs. control and pseudoletter vs. control contrasts. On the left, "glass brain" views: the bilateral activation occurring in the precentral gyri (vPMCinf) is circled in red. On the right, both patterns of activation are shown on a coronal brain slice $(y=5)$. (For interpretation of the references to colour in this figure legend, the reader is referred to the web version of the article.)

eral extra-striatal ventral visual areas and right parietal areas. This premotor activation was located more ventrally/medially than that specific to letters. The region where it occurred will be referred to below as the inferior ventral premotor cortex (vPMCinf), as opposed to the vPMCsup cluster previously described in the section dealing with the letter versus pseudoletter comparisons.

\subsection{Group comparison}

Direct statistical comparison between Lhrs and Rhrs was performed as follows: in a first step, we performed a random effects analysis, pooling together the 24 contrast images from the 13 Lhrs and 11 Rhrs (Longcamp et al., 2003) for the letters versus control contrast. This analysis revealed two large clusters of activation in the left and right premotor cortices. In a second step, we used MarsBar-SPM toolbox (Brett, Anton, Valbregue, \& Poline, 2002) to define two regions of interest (ROI) in the left and right vPMCsup. The ROI were defined as the common area between the volume of the cluster of activation and a sphere with a diameter of $10 \mathrm{~mm}$ centered on the statistical maximum of the vPMCsup cluster (Talairach coordinates $-54 ;-4 ; 44$ and 60; 0 ; 40 on the left and right-vPMC, respectively). This procedure allowed us to constrain the size of the ROI, in order to make it similar for the two hemispheres. In a third step, we extracted the mean contrast values for each subject within these ROI for the contrast letter versus pseudoletters: we therefore had two values per subject (i.e., one per ROI). Finally, we entered the mean individual contrast values into a two-way (hemisphere $\times$ handedness) ANOVA with repeated measures on the first factor and handedness as a between-subjects factor.

Table 2

Anatomical location, corresponding Brodmann area, lateralization, Talairach coordinates and Z-score for the activations common to letters and pseudoletters, as assessed by masking the letters vs. control and pseudoletters vs. control contrasts

\begin{tabular}{|c|c|c|c|c|c|c|c|c|}
\hline \multirow[t]{3}{*}{ Anatomical location } & \multicolumn{4}{|c|}{ Letter vs. control contrast } & \multicolumn{4}{|c|}{ Pseudoletters vs. control contrast } \\
\hline & \multicolumn{3}{|c|}{ Coordinates (mm) } & \multirow[t]{2}{*}{ Maximum Z-score } & \multicolumn{3}{|c|}{ Coordinates (mm) } & \multirow[t]{2}{*}{ Maximum $Z$-score } \\
\hline & $x$ & $y$ & $z$ & & $x$ & $y$ & $z$ & \\
\hline GOi/GOm (BA18/19/37) L & -40 & -60 & -12 & 5.55 & -38 & -86 & -4 & 5.14 \\
\hline GOi/GOm (BA18/19/37) R & 42 & -80 & -1 & 5.44 & 42 & -89 & 6 & 5.06 \\
\hline GOs (BA19) R & 30 & -76 & 31 & 4.06 & 32 & -76 & 31 & 3.75 \\
\hline GOm (BA19) L & -30 & -75 & 22 & 3.91 & -30 & -77 & 20 & 4.34 \\
\hline GPrC/GFi (BA6/44) R & 40 & 7 & 16 & 3.89 & 42 & 3 & 20 & 4.03 \\
\hline LPs (BA7) R & 32 & -57 & 60 & 3.83 & 32 & -55 & 58 & 3.72 \\
\hline GPoC (BA1/2) R & 44 & -23 & 38 & 3.63 & 50 & -19 & 40 & 4.32 \\
\hline GPrC (BA6) L & -44 & 3 & 29 & 3.60 & -46 & 4 & 31 & 3.72 \\
\hline
\end{tabular}

The values for the two contrasts are reported separately. Abbreviations: GOi, inferior occipital gyrus; GOm, middle occipital gyrus; GOs, superior occipital gyrus; GPrC, precentral gyrus; GFi, inferior frontal gyrus; LPs, superior parietal lobule; GPoC, postcentral gyrus. 
This analysis revealed a hemisphere by handedness interaction $(F(1,22)=9.45, p \leq 0.006)$, showing that the balance of activation between the two hemispheres was inverted for the two groups of subjects. Furthermore, we directly compared the two groups in each ROI using two $t$-tests. In the right premotor cortex, the letters/pseudoletters contrast was bigger in Lhrs than in Rhrs $\left(t_{22}=2.09, p<0.05\right)$. Conversely, in the Left premotor cortex, the contrast was bigger in Rhrs than in Lhrs but the difference failed to reach the level of significance $\left(t_{22}=1.73, p<0.10\right)$.

\section{Discussion}

The present study was designed to test the relationship between the motor-related activations elicited by passive visual perception of letters and the hand used to write, in order to confirm the existence of motor-perceptual interactions in letter reading. The results of a previous study (Longcamp et al., 2003) showed that an area in the left premotor cortex was selectively activated when Rhrs were observing letters but not pseudoletters. Since the same area was also activated when the subjects were actually writing the letters, we proposed that covert motor processes were triggered by the visual perception of letters. In this study in Lhrs, the activations observed will be discussed in detail when they are directly related to the main issue addressed in this paper. Other activations will be mentioned only for descriptive purposes, and will be briefly discussed insofar as they seem to be relevant to the question of the premotor activations.

\subsection{Brain areas specifically activated by letters}

In the present study, the Lhrs displayed a specific unilateral right ventral premotor activation (vPMCsup) during letter perception. Fig. 4 illustrates the right and the left vPMCsup clusters found, respectively, for Lhrs in this study and for Rhrs in the previous one: it shows that the two groups exhibited symmetrical clusters of premotor activation. This symmetry in the effects has further been evidenced by the results of the ANOVA comparing the LHrs and RHrs groups.

The occurrence of a unilateral, right, premotor activation specific to letter perception in Lhrs, opposite to the symmetrical left activation observed in Rhrs, rules out the possibility that this activation resulted from language processing. Since the phonological aspects of language are mediated by left hemispheric regions in most Lhrs (Knecht et al., 2000), the activation observed in the right premotor cortex is not likely to have reflected the phonological processing of letters. Instead, it can be explained by the involvement of motor programs for writing, which are unilaterally stored in the hemisphere contralateral to the writing hand. In the previous experiment, the participants were strictly right-handed and the premotor activation which occurred during letter perception was restricted to the left hemisphere. In the present experiment, the participants were Lhrs who had learned to write and actu-

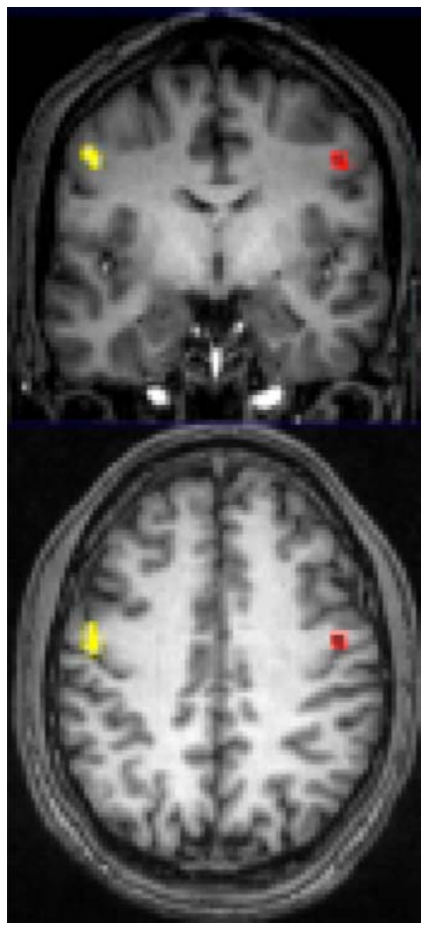

Fig. 4. Ventral premotor activation (vPMCsup) selectively occurring during letter perception in Lhrs (red) and Rhrs (yellow, see Longcamp et al., 2003 for details), shown on the same coronal and axial slices. The clusters were distinctly symmetrical but they differed in their spatial extent ( 84 voxels for Rhrs and 22 voxels for Lhrs), although the statistical values obtained in both cases were comparable ( $Z=4.37$ for Rhrs and $Z=4.11$ for Lhrs). (For interpretation of the references to colour in this figure legend, the reader is referred to the web version of the article.)

ally wrote with their left hand. We can therefore assume that in these subjects, the neural representation of writing movements was right-lateralized (Herron et al., 1979; Margolin, 1980; Margolin \& Binder, 1984; Rijntjes et al., 1999; Siebner et al., 2002). The vPMCsup cluster was less extensive in Lhrs than in Rhrs, however (Fig. 4). A lack of homogeneity in the Lhr group might be one explanation for this finding. As a matter of fact, Lhrs are known to display a variable pattern of functional organization in the brain (Hécaen \& Sauguet, 1971). Also, recent neuroanatomical studies have evidenced anatomical differences between Lhrs and Rhrs, especially a lack of asymmetry in the central (Amunts, Jäncke, Mohlberg, Steinmetz, \& Zilles, 2000) and precentral (Mangin et al., 2003) sulcal anatomy, and generally less asymmetry and/or greater variability in the amount of gray matter between the two hemispheres (Watkins et al., 2001). This may explain more convincingly why the statistical values of the effects observed were comparable, whereas the spatial extent of the activation differs. Despite the spatial normalization procedures used, the individual activations may have overlapped less than in Rhrs.

Lastly, some additional non-motor areas in the inferior frontal gyrus (BA 47), the middle temporal gyrus (BA 21) and the inferior parietal lobule (BA 40) were found here to be specifically activated during letter perception. All these acti- 
vations were right-lateralized; they are difficult to interpret, given the variability of language processing in left-handers. The inferior parietal activation for instance was previously described as part of the network involved in the execution of grasping movements (Rizzolatti \& Luppino, 2001). Note the absence of visual areas specialized in letter recognition, as previously suggested by several authors (Cohen et al., 2000; Polk et al., 2003). In fact, the existence of written languagespecialized visual areas has been challenged (Price \& Delvin, 2003).

\subsection{Brain areas commonly activated by letters and pseudoletters}

Surprisingly, in Lhrs we observed a bilateral involvement of the vPMCinf, another region of Brodmann's area 6, in response to the visual presentation of both letters and pseudoletters. This bilatereral activation differs from what was found in Rhrs for whom the only areas activated by the pseudoletters were the extrastriatal ventral visual areas (see Longcamp et al., 2003). Yet, the ventral premotor area is known to be involved in the movements performed to grasp, hold or reach objects (Johnson, Coltz, Hagen, \& Ebner, 1999; Rizzolatti \& Luppino, 2001). Thus, what can this activation be related to, since no specific motoric writing skills are associated with pseudoletters? Two possible interpretations can be proposed, based on the role of premotor areas in the perception of manipulable objects versus in their role in visuospatial processing. On one hand, a growing body of evidence indicates that ventral premotor neurons display additional visual properties, especially during object perception (Murata et al., 1997), and the results of several neuroimaging studies in humans showed that in Rhrs, the left vPMcx, together with the anterior intraparietal cortex (AIP), is consistently activated when observing, naming, storing in working memory or simply attending to images of manipulable objects such as tools (Chao \& Martin, 2000; Gerlach, Law, Gade, \& Paulson, 2000, 2002; Grèzes, Armony, Rowe, \& Passingham, 2003; Kellenbach, Brett, \& Patterson, 2003; Mecklinger, Gruenewald, Besson, Magnié, \& von Cramon, 2002). Strikingly, the coordinates of the activation clusters observed in all these studies match up well with the coordinates of the clusters observed in the present study during letter and pseudoletter perception (see Table 3). No motor program specific to pseudoletters is stored in memory, but it is possible that visual analysis of graphic stimuli would lead to "default" motor activations due to the implicit processes of categorizing them as "potentially writable" symbols.

On the other hand, it was recently suggested that this type of premotor activations can in fact be triggered by a broader

Table 3

Comparison between the coordinates of the vPMCinf cluster observed for letters and pseudoletters perception in the present study, and for objects perception or spatial tasks in other studies

\begin{tabular}{|c|c|c|}
\hline Study & Task & Coordinates vPMCinf \\
\hline \multicolumn{3}{|l|}{ Object perception studies } \\
\hline \multirow[t]{2}{*}{ Chao and Martin (2000) } & Viewing tools/animals & $-42 ; 6 ; 23$ \\
\hline & Naming tools/animals & $-50 ; 3 ; 25$ \\
\hline Mecklinger et al. (2002) & Images of manipulable objects held in working memory & $-46 ; 5 ; 31$ \\
\hline \multirow[t]{2}{*}{ Kellenbach et al. (2003) } & Judgments of function of manipulable/non-manipulable artefacts & $-54 ; 10 ; 28$ \\
\hline & Judgments of action associated with manipulable/function of non-manipulable artefacts & $-50 ;-2 ; 26$ \\
\hline Gerlach et al. (2000) & Categorization of artefacts/natural objects & $-64 ; 6 ; 20$ \\
\hline Gerlach et al. (2002) & Categorization of artefacts/natural objects & $-66 ; 6 ; 22$ \\
\hline Grèzes et al. (2003) & Viewing graspable object/baseline & $-44 ; 2 ; 30$ \\
\hline Mean & & $-52.0 ; 4.5 ; 25.6$ \\
\hline \multicolumn{3}{|l|}{ Spatial processing studies } \\
\hline \multirow[t]{2}{*}{ Schubotz and von Cramon (2001) } & Attending to the spatial configuration of geometrical forms & $-47 ; 4 ; 23$ (Left) \\
\hline & & 43; 4; 23 (Right) \\
\hline \multirow[t]{2}{*}{ Schubotz and von Cramon (2002) } & Predicting the size of a circle in an ordered sequence/color judgment & $-51 ; 3 ; 21$ (Left) \\
\hline & & $48 ; 8 ; 23$ (Right) \\
\hline \multirow[t]{2}{*}{ Lamm et al. (2001) } & Mental rotation of cubes & $-48 ; 4 ; 28$ (Left) \\
\hline & & $56 ; 4 ; 28$ (Right) \\
\hline Fink et al. (2003) & Line bisection judgement & 48; 14; 16 (Right) \\
\hline Richter et al. (2000) & Mental rotation of artificial 3D objects & Coordinates not reported \\
\hline \multirow[t]{2}{*}{ Mean } & & $-48.6 ; 3.7 ; 24$ (Left) \\
\hline & & 48.7; 7.5; 22.5 (Right) \\
\hline \multirow[t]{2}{*}{ Present study } & Viewing letters and pseudoletters/control & 40; 7; 16 (Right) \\
\hline & & $-44 ; 3 ; 29$ (Left) \\
\hline
\end{tabular}


range of artefacts, regardless of their specific manipulability (Gerlach et al., 2002; Kellenbach et al., 2003). Furthermore, activations in the lateral premotor cortex, very similar to those found for graphic forms in the present study (see Table 3) have been reported in other neuroimaging studies on tasks involving spatial analysis and mental rotation of $2 \mathrm{D}$ or $3 \mathrm{D}$ shapes (Lamm, Windischberger, Leodolter, Moser, \& Bauer, 2001; Richter et al., 2000; Schubotz \& von Cramon, 2001, 2002). Close analysis of the spatial conformation was particularly necessary to discriminate between letters and pseudoletters, since the pseudoletters used consisted of parts of real letters. It is therefore possible that the vPMCinf activation observed is related to some implicit mental rotation processes. This interpretation is strenghtened by the associated activation of the superior parietal lobule, a structure that has been found to be activated in tasks requiring a high level of visuospatial attention (Dong et al., 2000; Gitelman et al., 1999). It is impossible to determine which of the two interpretations best accounts for the present data. Moreover, the cognitive functions of the premotor areas are probably multiple and are the focus of a growing number of studies (Hanakawa et al., 2002; Rizzolatti et al., 2002). Further investigations will be necessary to clarify their precise involvement in general processing and categorization of graphic stimuli.

It is difficult to explain why a similar pattern of activation did not occur in the same situation in Rhrs. In fact, retrospective examination of Longcamp et al.'s data (2003) showed that passive pseudoletter viewing also activated the vPMCinf bilaterally in Rhrs, but not sufficiently to reach the statistical threshold. The difference between the two groups might therefore be more quantitative than qualitative. Cohen (1972) reported that Lhrs and Rhrs performed differently in a letter-classification task in particular, Lhrs showed fewer behavioral differences between the linguistic and spatial processing of letters. This finding tends to suggest that different strategies may be used by the two populations when analyzing graphic stimuli. Nevertheless, since only a few authors have focused on letter processing in general and even fewer have compared Lhrs and Rhrs in this respect, it is impossible to draw any definitive conclusions at present.

To conclude, the present findings show that passive visual perception of letters selectively activates a premotor area in the hemisphere contralateral to the hand usually performing writing movements. This supports the hypothesis that the visual perception of written language is somehow dependent on motor processes.

\section{Acknowledgements}

The research was funded by the French Research Ministry (A.C.I. 'Cognitique' COG73). Marieke Longcamp was supported by a fellowship from the French Research Ministry (A.C.I. 'Cognitique'). We thank Reyna Leigh Gordon for revising the English.

\section{References}

Amunts, K., Jäncke, L., Mohlberg, H., Steinmetz, H., \& Zilles, K. (2000). Interhemispheric asymmetry of the human motor cortex related to handedness and gender. Neuropsychologia, 38, 304-312.

Anderson, S. W., Damasio, A. R., \& Damasio, H. (1990). Troubled letters but not numbers: Domain specific cognitive impairments following focal damage in frontal cortex. Brain, 113, 749-766.

Brett, M., Anton, J. L., Valbregue, R., \& Poline, J. B. (2002). Region of interest analysis using an SPM toolbox. In Presented at the eighth international conference on functional mapping of the human brain (p. 16) (available on CD-Rom in NeuroImage).

Chao, L. L., \& Martin, A. (2000). Representation of manipulable manmade objects in the dorsal stream. NeuroImage, 12, 478-484.

Chen, R., Cohen, L. G., \& Hallet, M. (1997). Role of the ipsilateral motor cortex in voluntary movement. Canadian Journal of Neurological Sciences, 24, 284-291.

Cohen, G. (1972). Hemispheric differences in a letter classification task. Perception and Psychophysics, 11, 139-142.

Cohen, L., Dehaene, S., Naccache, L., Lehericy, S., Dehaene-Lambertz, S., Henaff, M. A., et al. (2000). The visual word form area: Spatial and temporal characterization of an initial stage of reading in normal subjects and posterior split-brain patients. Brain, 123, 291307.

Dong, Y., Fukuyama, H., Honda, M., Okada, T., Hanakawa, T., Nakamura, $\mathrm{K}$., et al. (2000). Essential role of the right superior parietal cortex in Japanese kana mirror reading: An fMRI study. Brain, 123(Pt 4), 790-799.

Fink, G. R., Marshall, J. C., Weiss, P. H., Stephan, T., Grefkes, C., Shah, N. J., et al. (2003). Performing allocentric visuospatial judgments with induced distortion of the egocentric reference frame: An fMRI study with clinical implications. NeuroImage, 20, 1505-1517.

Flores d'Arcais, G. B. (1994). Order of strokes writing as a cue for retrieval in reading Chinese characters. European Journal of Cognitive Psychology, 6, 337-355.

Friston, K. J., Holmes, A. P., Poline, J. B., Grasby, P. J., Williams, S. R. C., Frackowiak, R. S. J., et al. (1995). Analysis of fMRI time-series revisited. Neurolmage, 2, 45-53.

Friston, K. J., Price, C. J., Fletcher, P., Moore, C., Frackowiak, R. S. J., \& Dolan, R. J. (1996). The trouble with cognitive subtraction. Neurolmage, 4, 97-104.

Gerlach, C., Law, I., Gade, A., \& Paulson, O. B. (2000). Categorization and category effects in normal object recognition: A PET study. Neuropsychologia, 38, 1693-1703.

Gerlach, C., Law, I., Gade, A., \& Paulson, O. B. (2002). The role of action knowledge in the comprehension of artifacts-A PET study. NeuroImage, 15, 143-152.

Gitelman, D. R., Nobre, A. C., Parrish, T. B., LaBar, K. S., Kim, Y., Meyer, J. R., et al. (1999). A large-scale distributed network for covert spatial attention. Further anatomical delineation based on stringent behavioural and cognitive controls. Brain, 122, 1093-1106.

Grèzes, J., Armony, J. L., Rowe, J., \& Passingham, R. E. (2003). Activations related to "mirror" and "canonical" neurons in the human brain: An fMRI study. NeuroImage, 18, 928-937.

Hanakawa, T., Honda, M., Sawamoto, N., Okada, T., Yonekura, Y., Fukuyama, H., et al. (2002). The role of rostral Brodmann area 6 in mental operation tasks: An integrative neuroimaging approach. Cerebral Cortex, 12, 1157-1170.

Hécaen, H., \& Sauguet, J. (1971). Cerebral dominance in left-handed subjects. Cortex, 7, 19-48.

Herron, J., Galin, D., Johnstone, J., \& Ornstein, R. E. (1979). Cerebral specialization, writing posture, and motor control of writing in lefthanders. Science, 205, 1285-1289.

Johnson, M. T. V., Coltz, J. D., Hagen, M. C., \& Ebner, T. J. (1999). Visuomotor processing as reflected in the directional discharge of premotor and primary motor cortex neurons. Journal of Neurophysiology, $81,875-894$. 
Katanoda, K., Yoshikawa, K., \& Sugishita, M. (2001). A functional MRI study on the neural substrates for writing. Human Brain Mapping, 13, 34-42.

Kato, C., Isoda, H., Takehar, Y., Matsuo, K., Moriya, T., \& Nakai, T. (1999). Involvement of motor cortices in retrieval of kanji studied by functional MRI. Neuroreport, 10, 1335-1339.

Kellenbach, M. L., Brett, M., \& Patterson, K. (2003). Actions speak louder than functions: The importance of manipulability and action in tool representation. Journal of Cognitive Neuroscience, 15, 30-46.

Knecht, S., Drager, B., Deppe, M., Bobe, L., Lohmann, H., Floel, A., et al. (2000). Handedness and hemispheric language dominance in healthy humans. Brain, 123, 2512-2518.

Lamm, C., Windischberger, C., Leodolter, U., Moser, E., \& Bauer, H. (2001). Evidence for premotor cortex activity during dynamic visual imagery from single trial functional magnetic resonance imaging and slow cortical potentials. NeuroImage, 14, 268-283.

Longcamp, M., Anton, J. L., Roth, M., \& Velay, J. L. (2003). Visual presentation of single letters activates a premotor area involved in writing. Neurolmage, 19, 1492-1500.

Mangin, J. F., Régis, J., Rivière, D., Cointepas, Y., Ochiai, T., Papadopoulos-Orfanos, D. L., et al. (2003). Structure based morphometry of cortical folding correlates of handedness. In Presented at the ninth international conference on functional mapping of the human brain (p. 19) (available on CD-Rom in NeuroImage).

Margolin, D. I. (1980). Right hemisphere dominance for praxis and left hemisphere dominance for speech in a left-hander. Neuropsychologia, 18, 715-719.

Margolin, D. I., \& Binder, L. (1984). Multiple component agraphia in a patient with atypical cerebral dominance: An error analysis. Brain and Language, 22, 26-40.

Mecklinger, A., Gruenewald, C., Besson, M., Magnié, M. N., \& von Cramon, D. Y. (2002). Separable neuronal circuitries for manipulable and non-manipulable objects in working memory. Cerebral Cortex, 12, 1115-1123.

Menon, V., \& Desmond, J. E. (2001). Left superior parietal cortex involvement in writing: Integrating fMRI with lesion evidence. Cognitive Brain Research, 12, 337-340.

Murata, A., Fadiga, L., Fogassi, L., Gallese, V., Raos, V., \& Rizzolatti, G. (1997). Object representation in the ventral premotor cortex (area F5) of the monkey. Journal of Neurophysiology, 78, 2226-2230.

Oldfield, R. C. (1971). The assessment and analysis of handedness. Neuropsychologia, 9, 97-113.

Paillard, J. (1991). Motor and representational framing of space. In J. Paillard (Ed.), Brain and space (pp. 163-182). Oxford: Oxford University Press.

Paulesu, E., Frith, C. D., \& Frackowiak, R. S. J. (1993). The neural correlates of the verbal component of working memory. Nature, 362, $342-345$.

Polk, T. A., Stallcup, M., Aguirre, G. K., Alsop, D. C., D’Esposito, M., Detre, D. A., et al. (2003). Neural specialization for letter recognition. Journal of Cognitive Neuroscience, 14, 145-159.
Price, C. J., \& Delvin, J. T. (2003). The myth of the visual word form area. NeuroImage, 19, 473-481.

Price, C. J., Wise, R. J. S., \& Frackowiak, R. S. J. (1996). Demonstrating the implicit processing of visually presented words and pseudowords. Cerebral Cortex, 6, 62-70.

Price, C. J., Wise, R. J. S., Watson, J. D. G., Patterson, K., Howard, D., \& Frackowiak, R. S. J. (1994). Brain activity during reading: The effects of exposure duration and task. Brain, 117, 12551269.

Richter, W., Somorjai, R., Summers, R., Jarmasz, M., Menon, R. S., Gati, J. S., et al. (2000). Motor area activity during mental rotation studied by time-resolved single-trial fMRI. Journal of Cognitive Neuroscience, 12, 310-320.

Rijntjes, M., Dettmers, C., Büchel, C., Kiebel, S., Frackowiak, R. S. J., \& Weiller, C. (1999). A blueprint for movement: Functional and anatomical representations in the human motor system. The Journal of Neuroscience, 19, 8043-8048.

Rizzolatti, G., Fogassi, L., \& Gallese, V. (2002). Motor and cognitive functions of the ventral premotor cortex. Current Opinion in Neurobiology, 12, 149-154.

Rizzolatti, G., \& Luppino, G. (2001). The cortical motor system. Neuron, 31, 889-901.

Roeltgen, D. (1985). Agraphia. In K. M. Heilman \& E. Valenstein (Eds.), Clinical neuropsychology (pp. 75-96). Oxford University Press: New York.

Schubotz, R. I., \& von Cramon, D. Y. (2001). Functional organisation of the lateral premotor cortex: fMRI reveals different regions activated by anticipation of object properties, location and speed. Cognitive Brain Research, 11, 97-112.

Schubotz, R. I., \& von Cramon, D. Y. (2002). Predicting perceptual events activates corresponding motor schemes in lateral premotor cortex: An fMRI study. NeuroImage, 15, 787-796.

Siebner, H. R., Limmer, C., Peinemann, A., Drzezga, A., Bloem, B. R., Schwaiger, M., et al. (2002). Long-term consequences of switching handedness: A positron emission tomography study on handwriting in "converted" left-handers. Journal of Neuroscience, 22, 28162825.

Smith, E. E., \& Jonides, J. (1999). Storage and executive processes in the frontal lobe. Science, 283, 1657-1661.

Talairach, J., \& Tournoux, P. (1988). Co-planar stereotaxic atlas of the human brain. New York: Thieme.

van Galen, G. P. (1991). Handwriting: Issues for a psychomotor theory. Human Movement Science, 10, 165-191.

Viviani, P., \& Stucchi, N. (1992). Motor-perceptual interactions. In J. Requin \& G. Stelmach (Eds.), Tutorials in motor behavior II (pp. 229-248). Amsterdam: Elsevier North-Holland.

Watkins, K. E., Paus, T., Lerch, J. P., Zijdenbos, A., Collins, D. L., Neelin, P., et al. (2001). Structural asymmetries in the human brain: A voxel-based statistical analysis of 142 MRI scans. Cerebral Cortex, $11,868-877$. 